\title{
Effect of rich Selenium Mushroom Polysaccharides on Hyperlipidemia Metabolism and Lipoprotein Lipase Activity of Tests Mice
}

\author{
Yue Li-hong ${ }^{1}$, Wang Yu-jun ${ }^{1}$, Yu Jing-ni ${ }^{1}$ and Zhang Yue-hua ${ }^{2, a}$ \\ ${ }^{1}$ College of Life Science, Jiamusi University, China \\ ${ }^{2}$ College of Science, Jiamusi University, China
}

\begin{abstract}
The Selenium-Polysaccharides from Rich selenium Chemical explicitly defined the Edible Fungus (Se-EF) in Boiling water extraction by alcohol precipitation, analysis indicated that the there were main ingredient comprises xylose, dextrose, rhamnose, galactose, and arabinose. First investigate lipids Metabolic and lipoprotein lipase (LPL) activated in the selenium-rich -EF rich in cholesterol and prospects by feeding rich foods (PRD). The purpose of this study is to explore the prospect 1 st fed cholesterol-enriched diet (PRD) of KM mice selenium -EF on cholesterol metabolism and lipoprotein lipase (LPL) activated of antioxidant capacity. In addition, studies of the relationship between atherosclerosis and total antioxidant capacity index (AI) and LPL activity (TAC). The results showed that selenium polysaccharide significantly reduce TC serum total cholesterol and LDL-C concentrations comparatively in with the CK control groups. Furthermore, orally administered of polysaccharide selenium notable enhance pay, lipoprotein lipase activity and reduce MDA levels, and artificial intelligence. These results indicate that the beneficial affects of selenium on -EF hypercholesterolemia precautions. In addition, oral administration of polysaccharide selenium notable enhance TAC and LPL activity in the meantime reduce MDA levels, the same as AI. These results indicate that Se-EF has a beneficial effect on the prevention of hyperlipidemia. These results indicate that the beneficial affects of selenium on -EF hypercholesterolemia precautions.
\end{abstract}

\section{Introduction}

The numbers of people suffering from hyperlipidemia, cardiovascular illness, rectally cancer was straight up in developing China. In medical, surgical, and endovascular treatment of tremendous progress, the significant number of intervention Implementation Constantly to grow each year. While Fatty and high protein composition is the main factors of lipid metabolism. It should not be ignored without food [1] skimmed part of. Statistics show that there is black fungus as dietary supplements May effectively reduce the incidence of cardiovascular and cerebrovascular. However, there are few papers on the direction of hyperlipidemia, atherosclerosis index and the lipoprotein lipase so far. In the past ten years, Lots of attention concentrated the metabolism of and atherosclerosis. In the past decade, A lot of attentions has concentrate on the affect of LPL on lipid cholesterol and atherogenesis [2]. Inhibition of LPL activity may Enhance TG and Reduction HDL and cholesterol (HDLC) concentration. This theory is well known, it is assumed in the oxidation- reduction state. Changes due to LPL activity change. Therefore, in this study, it is assumed to that antioxidants may directly or indirectly affect LPL activity by varying oxidation and reduction state as the cells to studied the total antioxidant- reducing ability, cholesterol activity, MDA level, As well as other indicators, -EF effects And high body mass trumpet, the levels of Intake of high-fat foods, in vivo food conversion efficiency, lipid and KM mice atherosclerosis index [3]. Moreover, the Ratio of TC-(HDL-C) and (HDL-C) is the formula is $\mathrm{Al}$, has been the risk of cardiovascular illness, like increased hazard in proportional to the increase in the proportion of directly related. For this perspective, the association between artificial intelligence and LPL activity and overall anti-oxidation ability (TAC) were evaluated between [4]. Therefore, the conclusion will promote know- ledge of black fungus cardiovascular benefit, and to explore its prevention in functional foods and health products for cardiovascular disease / therapeutic applications.

\section{Materials and methods}

\subsection{Review stage}

The method of Se-A. Auricular polysaccharide material was preparation. The basic components of the Se-A. Auricular material corresponding has been measurement. The total dietary fiber content by enzymatic method and gravimetric method has been measuring. The determination of total fat content was gas chromatography technique. Determination of nitrogen

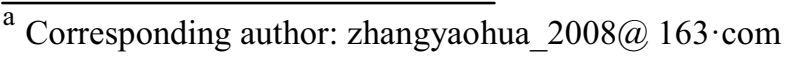


performed done according to the method established by using conventional methods using Bovine serum albumin (BSA) as Reference Sample. The nitrogen is converted to protein mass obtained, the correlation coefficient was calculated 6.25 [5]. The samples of A. auricular artificial was cultivation, in BOBABU Biotech Co. Ltd., Jiamusi City, Heilongjiang Province, China. A. auricular samples at $60{ }^{\circ} \mathrm{C}$, initial oven 2 hours, and on the ground with electric grinding mill and passed through a 40 meshes and 60 mesh sieves. A. auricular containing lyophilized carbo- hydrates (grams per $100 \mathrm{~g}$ dry weight) of 80 (comprises 54 grams overall dietary fiber), 8.0g Raw protein, fat $1.3 \mathrm{~g}$, ashes 8.7 . Powder samples $200.0 \mathrm{~g}$ in $3.0 \mathrm{~L}$ alcohol extraction for 48 hours at room temperature, low molecular weight fat components and filtration removed through water-soluble substances Xin-Hua 2th paper to remove small molecular weight components of the lipid composition and insoluble's.

This process is performed three times. The filtered product was dissolved in distilled water and transferred to a 3 liters reflux apparatus purified at $100^{\circ} \mathrm{C}$ condition for repeated two times. Extracts were filtered and concentrated under reduced pressure device, combined with precipitate was extracted with alcohol to obtain the polysaccharide was repeated 4 times. Obtained insoluble material was collected by centrifuge, washed by alcohol, freeze-dried to remove the volatile components, the crude polysaccharide obtained, namely $47.8 \mathrm{~g}$, then powdery product with an electric grinder for subsequent research. The resulting product collect by centrifugation, and washed with alcohol, ion exchange technology and lowtemperature drying, to obtain $48.7 \mathrm{~g}$ of crude sugar, and then with an electric grinder to grind into a powder.

\subsection{Composition analysis}

The Total carbohydrate contents in Calculated using the 3, 5-dinitrosalicylic acid as internal reference standard phenol-sulfuric acid method. The glucuronide is as a standard sample, using the colorimetric determination of total urinate. Sample ashes conditions are: Step 1: $600^{\circ} \mathrm{C}$, 1 hour. Step 2: $800{ }^{\circ} \mathrm{C}$ constant weight. 4 hours. Determination of the nitrogen content was in accordance with the foregoing method.

\subsection{Animal experiment}

All of standardized male KM-Mice (Experimental Animal Center of the 2th Harbin hospital, China) 5 days before the experiment domestication were being randomly divided into experimental group. At a temperature of $23 \pm 2^{\circ} \mathrm{C}$ and $55 \pm 5 \%$ humidity conditions, alone in a separate cage with 12 hours of light and shade in breeding mice (form 8:00 to 20:00), at $24 \pm 1{ }^{\circ} \mathrm{C}$ of and at the temperature of $60 \pm 4 \%$ humidity, the timing weighed. In the feeding period, In the food (as shown in Table 1) and Water and food without restrictions, free to feed $5 \mathrm{wks}$, The experimental KM-mice were divided into three groups of 12, The first group named control group (NG), The second group as high cholesterol group (MG), and The third group is the self-treatment group (EG). NG of KM mice fed normal diet, MG and EG of KM mice were fed cholesterol-rich foods diet.

Table 1. Chemical ingredients of sample diets (In per100 g dry weight).

\begin{tabular}{|c|c|c|}
\hline \multirow{2}{*}{ Compositions } & \multicolumn{2}{|c|}{ Content } \\
\hline & $\mathrm{RD}$ & PRD \\
\hline Carbohydrate & 58 & 49 \\
\hline Protein & 21.4 & 21.6 \\
\hline Fiber & 4.9 & 4.9 \\
\hline Fat & 4.2 & 4.2 \\
\hline$P$ & 1.2 & 1.2 \\
\hline Ash & 8 & 8 \\
\hline Sodium cholic acid & --- & 0.2 \\
\hline Cholesterol & --- & 1 \\
\hline Lard & -- & 8.7 \\
\hline $\mathrm{Ca}$ & 2.7 & 1.9 \\
\hline
\end{tabular}

Experimental animal feed ingredients shown in Table 1. During the eight weeks, EG DEF of KM mice were administered by oral gavage using $120 \mathrm{mg} / \mathrm{kg} /$ day of body weight once a day, MG, NG and orally administered an equal volume of distilled water as a control. Experimental animal body weight was measured in accordance with S-EF fraction oral gavage dose alternate days. Daily food intake measurement ibid. Blood was collected and analyzed every week [6]. Efficiency of food intake of laboratory mice is calculated based on the following formats: Daily weight gain / daily food intake $\times 100$. Experimental procedures are performed according to the experimental animals manual Harbin Medical University. (Harbin, China).

\subsection{Experimental animal serum samples collected}

Orbital venous plexus blood samples KM mice without anesthesia, quickly taken from sterile heparin-containing capillary, operating respectively in 4,6 and 8 weeks, respectively. The experimental animals were transferred to a sterile $10 \mathrm{ml}$ blood plastic tubes, $37^{\circ} \mathrm{C}$ in $15 \mathrm{~min}$, centrifuged for 8 minutes at $3600 \mathrm{rpm}$ condition, the serum was separated. The serum samples of experimental animals placed in a refrigerator at $-20^{\circ} \mathrm{C}$, for serum total cholesterol, LDL-C, HDL-C and triglyceride concentrations were measured serum lipids, these indicators by enzyme kit methods.

\subsection{TAC, LPL and Lipid per-oxidation determinations}

TAC, LPL and Lipid per-oxidation determinations were correlation detection kit purchased from Shanghai enzyme- linked Biotech Company. All operational requirements and processes are measured in accordance with the instructions 


\subsection{Statistical analysis of experimental data}

Statistical experimental data to each group $12 \mathrm{KM}$ mice a computing unit for \pm standard deviation were calculated. The design of the experimental data was using a one-way analysis of variance approach to statistical analysis in routine experimentation [7]. TAC relationship between $\mathrm{AI}$ and LPL activity by the Pearson product moment correlation coefficient was clarify and analyzed by SPSS16.3.

\section{Results}

\subsection{The chemical composition of the experimental samples of polysaccharide}

Chemical test samples S-EF compositions are listed in Table 2, composed of glucose, rhamnose, uronic acids, xylose, and sulfate were the main composition besides smaller amounts of arabinose, galactose, and mannose etc. Furthermore, there is a lot of money in the amount of ash product, which is caused by byproducts of sulfate and polysaccharides ironic ion binding composition. These various proteins may form with sulfated polysaccharide conjugate, was stable structure.

Table 2.Chemical ingredient of polysaccharide from Se-A. Fungus

\begin{tabular}{|c|c|}
\hline Ingredient & $\begin{array}{c}\text { Content } \\
\text { Component }\end{array}$ \\
\hline Arabinose & 0.2 \\
\hline Ash & 20.4 \\
\hline Mannose & 1.4 \\
\hline Rhamnose & 13.4 \\
\hline Sulfate & 15.7 \\
\hline Total sugars & 41.5 \\
\hline Galactose & 0.7 \\
\hline Glucose & 12.6 \\
\hline Nitrogen & 1.8 \\
\hline Se & $2.63 \times 10^{4}$ \\
\hline U. acids & 19.7 \\
\hline Xylose & 15.3 \\
\hline
\end{tabular}

Table 3.Changes of weight and food intake of KM-Mice in tested periods.

\begin{tabular}{|c|c|c|c|}
\hline Test Project & EG & MG & NG \\
\hline $\begin{array}{c}\text { Initial body wt } \\
\text { (g/mouse) }\end{array}$ & $22 \pm 2.2$ & $22 \pm 2.4$ & $24 \pm 2.0$ \\
\hline $\begin{array}{c}\text { Ultimate body wt } \\
\text { (g/mouse) }\end{array}$ & $41 \pm 2.1$ & $41 \pm 2.0$ & $42 \pm 1.8$ \\
\hline Weight gain \\
(g/mouse/d) & $0.3 \pm$ & $0.5 \pm 0.07$ & $0.3 \pm$ \\
\hline $\begin{array}{c}\text { Food intake } \\
\text { (g/mouse/d) }\end{array}$ & 0.06 & & 0.04 \\
\hline Feeding efficiency & $11.1 \pm 0.6$ & $2.9 \pm 0.8$ & $3.6 \pm$ \\
(\%) & 1.4 & $13.1 \pm 0.9$ & $10.8 \pm$ \\
\hline \multicolumn{3}{|c|}{ Note: $(\mathbf{P}<\mathbf{0 . 0 5})$} \\
\hline
\end{tabular}

\subsection{The efficiency in experimental animals body weight, food intake and feed}

Experimental data show that those animals in Table 3, the normal control group and two experimental groups no significant difference in weight indicators, the same as The levels of daily food intake of the EG and MG diet groups. The levels of feeding efficiency of the EG and the MG were 11.2 and $12.3 \mathrm{~g}$, respectively, compared with normal control group is relatively higher.

Table 4. Influences of S-EF on serum lipids in NG, MG and EG.

\begin{tabular}{|c|c|c|c|c|}
\hline \multicolumn{2}{|c|}{ Serum lipids } & NG & MG & EG \\
\hline \multirow{4}{*}{ TG } & $\mathrm{W} 2$ & $1.77 \pm 0.15$ & $1.38 \pm 0.15 b$ & $1.68 \pm 0.12 \mathrm{a}$ \\
\hline & W4 & $1.73 \pm 0.12$ & $1.39 \pm 0.16 \mathrm{~b}$ & $1.66 \pm 0.16 \mathrm{a}$ \\
\hline & W6 & $1.76 \pm 0.14$ & $1.41 \pm 0.19 b$ & $1.69 \pm 0.13 \mathrm{a}$ \\
\hline & W8 & $1.78 \pm 0.12$ & $1.38 \pm 0.17 b$ & $1.67 \pm 0.15 \mathrm{a}$ \\
\hline \multirow{4}{*}{ TC } & W2 & $5.07 \pm 0.48$ & $10.93 \pm 0.72 b$ & $7.09 \pm 0.11 \mathrm{ab}$ \\
\hline & W4 & $5.11 \pm 0.45$ & $11.75 \pm 0.83 b$ & $\begin{array}{c}7.65 \pm \\
0.13 \mathrm{ab}\end{array}$ \\
\hline & W6 & $5.14 \pm 0.52$ & $11.32 \pm 0.56 b$ & $\begin{array}{l}8.18 \pm \\
0.16 a b\end{array}$ \\
\hline & W8 & $5.09 \pm 0.49$ & $11.58 \pm 0.76 b$ & $\begin{array}{l}8.23 \pm \\
0.21 \mathrm{ab}\end{array}$ \\
\hline \multirow{4}{*}{$\begin{array}{c}\text { HDL- } \\
\text { C }\end{array}$} & W2 & $3.85 \pm 0.59$ & $4.11 \pm 0.71$ & $\begin{array}{c}3.96 \pm \\
0.98 b\end{array}$ \\
\hline & W4 & $3.76 \pm 0.61$ & $4.24 \pm 1.13 b$ & $\begin{array}{c}4.08 \pm \\
1.12 b\end{array}$ \\
\hline & W6 & $3.78 \pm 0.76$ & $4.38 \pm 1.18 b$ & $\begin{array}{c}4.12 \pm \\
1.04 \mathrm{~b}\end{array}$ \\
\hline & W8 & $3.82 \pm 0.83$ & $4.35 \pm 1.14 b$ & $\begin{array}{c}4.19 \pm \\
1.26 \mathrm{~b}\end{array}$ \\
\hline \multirow{4}{*}{$\begin{array}{c}\text { LDL- } \\
\text { C }\end{array}$} & W2 & $0.35 \pm 0.04$ & $0.49 \pm 0.09 b$ & $0.39 \pm 0.05 \mathrm{a}$ \\
\hline & W4 & $0.38 \pm 0.07$ & $0.52 \pm 0.12 b$ & $0.42 \pm 0.07 \mathrm{a}$ \\
\hline & W6 & $0.37 \pm 0.06$ & $0.55 \pm 0.08 b$ & $\begin{array}{l}0.48 \pm \\
0.08 \mathrm{ab}\end{array}$ \\
\hline & W8 & $0.37 \pm 0.12$ & $0.54 \pm 0.11 b$ & $\begin{array}{l}0.48 \pm \\
0.09 \mathrm{ab}\end{array}$ \\
\hline
\end{tabular}

Note: (A: $P<0.05$ Versus MG; B: $P<0.05$ Versus NG).

\subsection{Effect of S-EF in lipoprotein and serum-lipid concentrations}

After experimental samples intervention for 2 week, the serum concentrations of HDL-C LDL-C, and TC, the Raise obviously about on, the same as TG content in the same manner. As shown in Table 4. The serum-lipid profile Remained relatively constant in the tested lasted in $\mathrm{MG}$ and $\mathrm{NG}$ groups. In the medium term, S-EF interventions can significantly reduce LDL-C and TC levels; there is relatively slight elevation over time more than eight weeks during the rearing period. However, significant differences were not observed between $\mathrm{MG}$ and $\mathrm{EG}$ in the concentration of $\mathrm{C}$ and HDL. In addition, 
S-EF can regulate the content of TG to normal level, which shows subtle difference with NG.

\subsection{Influences of S-EF levels Intervention in LPL activity}

The Effects of s-EF Intervention in LPL activity Illustrate the Function of S-EF in that LPL activity of PRD-fed test group as shown in Fig. 1. LPL activity of PRD-fed KM mice in the serum was significantly reduced. S-EF treatment significantly inhibited PRD induced negative changes, NG comparison with experimental data show that significant differences do not exist. However, these data suggest TAC data relating to indicators associated with $\mathrm{AI}, \mathrm{AI}$ is directly related to the oxidative stress.

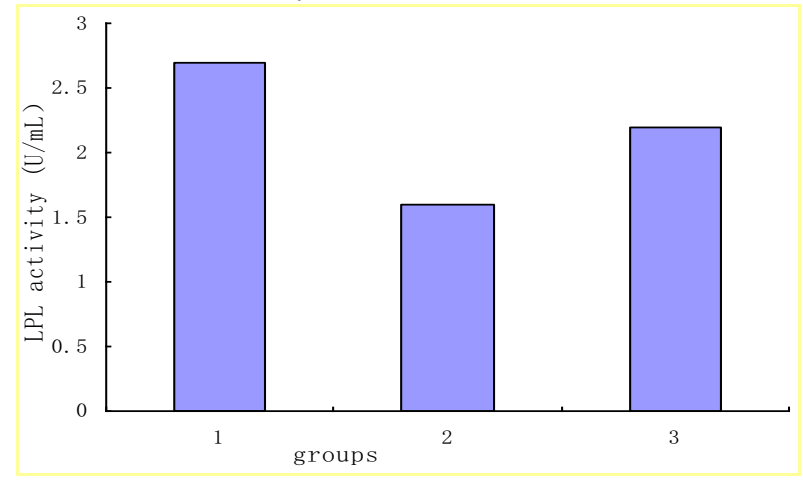

Figure 1. Compare of EF in LPL function of PRD-fed groups

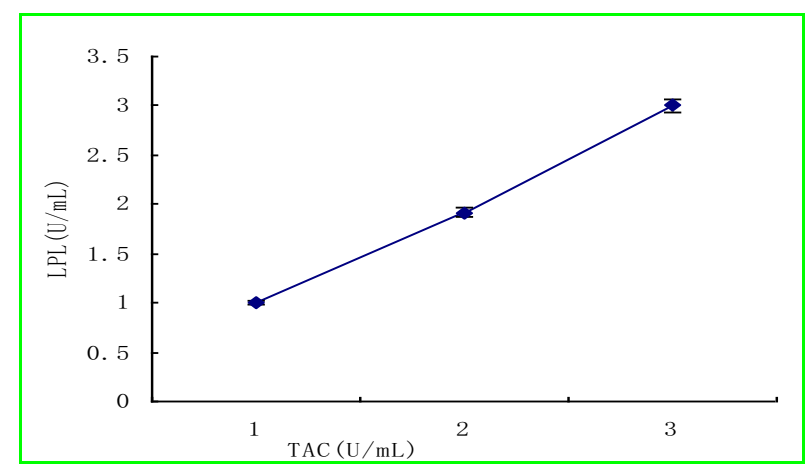

Figure 2. Correlation between TAC and atherosclerotic index.

\subsection{Correlation between TAC and the Al}

The relationship between AI and TAC was exposed by correlation analysis (Fig. 1). The analysis indicate a inverse correlation (Fig. 2). As a result, due to lower oxidation resistance, AI was relatively high in the series.

\subsection{Activity of compare relationship between TAC and LPL}

In Fig. 1, the correlation between the activity of LPL and TAC is represented. Positive correlation between LPL and TAC activity was discover. The correlation of TAC and LPL activity demonstrate that the change of redox state played anpositive function in the transform of LPL activity.

\subsection{Effect of S-EF for MDA content}

To investigate whether oxidative stress occurs in NG-fed KM mice, to satisfy of MDA was measured in these laboratory animals. The tests dates are disclosed in Table 5 , to compare in $\mathrm{MG}$, there was a remarkable reduces to compare MDA Gradation with the serum indicators of EG.

Table 5. Effect of EF on the AI of PRD-fed groups compared with control group. groups NG, MG and EG

\begin{tabular}{cccc}
\hline Groups & NG & MG & EG \\
\hline \multirow{2}{*}{$\mathrm{AI}$} & $0.35 \pm$ & $1.76 \pm 0.21 \mathrm{~b}$ & $0.39 \pm$ \\
& 0.11 & & $0.07 \mathrm{ab}$ \\
$\mathrm{TAC}(\mathrm{U} / \mathrm{mL})$ & $2.54 \pm$ & $0.89 \pm 0.04 \mathrm{~b}$ & $2.21 \pm$ \\
& 0.09 & & $0.05 \mathrm{ab}$ \\
$\mathrm{LPL}(\mathrm{U} / \mathrm{mL})$ & $2.79 \pm$ & $1.29 \pm 0.09 \mathrm{~b}$ & $2.29 \pm$ \\
& 0.15 & $29.89 \pm$ & $0.12 \mathrm{ab}$ \\
$\mathrm{MDA}(\mathrm{nmol} / \mathrm{mL})$ & $9.99 \pm$ & $2.38 \mathrm{~b}$ & $1.21 \mathrm{a}$ \\
& 0.89 & $\mathbf{0 . 0 5}$ about MG; $\mathbf{~} \mathbf{P}<\mathbf{0 . 0 5}$ about $\mathbf{N G}$
\end{tabular}

\section{Discussion}

S-EF Constraints therapy the downside diversification produced by PRD. it is consistently recognized that depression the level of high serum cholesterol administer to the prevention of atherosclerosis. National will take additional measures to decrease the concentration of serum cholesterol through adjusting High cholesterol food structure in their daily lives. Latest research achievements on human and animal subjects were indicated that a Diet increased fungus and other fungi foods have significantly lower blood lipids clinical effect [8]. These tests investigated the influence East Asian food (black fungus) polysaccharide on serum and blood lipid of mice is very clear intervention. In the research, the levels of HDL-C and LDL-C in serum of MG were differs notable with NG. This may be a stress response caused by high cholesterol levels in KM mice, which the correlation is significantly higher than of serum HDL-C and LDL-C. Which the Metabolic homeostasis is altered, the concentration of HDL-C and LDL-C in serum can be severely altered to form a hypercholesterolemia, even atherosclerotic sis.

This research suggests high cholesterol food intake and weight gain is insignificant in the diet were significantly different from that of the diet fed mice. The results showed that the contents of S-EF and TC $(\mathrm{P}<$ $0.05)$ in serum were significantly lower than that in serum LDL-C $(\mathrm{P}<0.05)$.

It is also indicated that s-EF decreased serum TC may be due to decreased serum LDL-C. Results were consistent with previous findings. Based on the above research foundation, we think that $\mathrm{S}-\mathrm{EF}$ is the main component of to reduce the serum TC concentration [9]. In addition, the balance of triglycerides also has the aforementioned studies show significantly increased risk of coronary artery disease and other heart diseases. The test data reveals that the activity of LPL TAC was 
positively correlated with, and I was a negative correlation TAC. Correspondingly it is whereas the AI was negatively correlated with TAC. This may be an important role in the reduction of AI. Lipoprotein has been a glycoprotein Change intervention fat into the peripheral tissue energy [10]. It frees fatty acid separation from TGs current in chylomicrons and unusual small density lipoprotein. Our research represents that LPL activity possibly was changed from the S-EF. In addition, according to our results, clear liquid level of MDA in magnesium was higher than that in NG. It indicates that the hypercholesterolemia may increase oil peroxide metabolism. It comes down to the hyper -cholesterolemia perhaps raise the small pieces, polymorphic nuclear cells, white blood cells and endothelial cells cholesterol content [11]. Thus MDA production may initiate a series of reactions and increase the oil peroxide route [12]. Our results indicated that $\mathrm{S}-\mathrm{EF}$ oral prevented perhaps that hypercholesterolemia may cause the elevation of MDA. The conclusion results showed that, the whole weight of $120 \mathrm{mg} / \mathrm{kg} / \mathrm{d} \mathrm{S}-\mathrm{EF}$ in along with the increment of diet management major stimulus in LPL activity and TAC, in which PRD feed KM Mice significantly reduced the supernatant of TC and LDL-C concentration and suppress the progress of atherosclerosis.

\section{Conclusions}

Comprehensive experimental data revealed that $120 \mathrm{mg} /$ $\mathrm{kg} /$ day of body weight target food intervention S-EF remarkable lower serum total cholesterol and LDL-C concentrations and inhibition of atherosclerosis progression PRD KM mice fed with the increase significantly stimulate LPL activity and TAC. However, it's about the publication of the S-EF research is quite limited. Moreover, anti S-EF effect of antihypercholesterolemia accurate effective components, site and mechanisms have not yet to be determined. Therefore, more research is needed to clarify the S-EF products on anti-hypercholesterolemia and atheroprotection further.

\section{Acknowledgment}

This work was financially supported by science and technology research programs of Jiamusi University of key research projects, Number: 12Z1201513.

\section{References}

1. E. Guillamón, A. García-Lafuente, M. Lozano, Edible mushrooms. Fitoterapia 81, 7 (2010)

2. R. L. Yang, G. Le, A. Li, J. Zheng, Y Shi. Nutrition. 22, 11, (2006)

3. E. S. Ferreira, M. A. Silva, A. Demonte, V. A. Neves Soy. Journal of medicinal food. 14,1-2 (2011)

4. R. Di Benedetto, L. Attorri and F. Chiarotti. Journal of agricultural and food chemistry. 58,9 (2010)

5. G. F. Pauli, T. Gödecke, B. U. Jaki and D. C. Lankin. Journal of natural products. 75,4(2012)

6. C. M. Waggoner. California State University. Long Beach (2011)

7. R. B. Matthew, B. T. Jewell, M. F. Anne, H. Jason, M. H. David, L. T. Benzinger, J. C. Morris and M. A. Beau. Neurobiology of aging, 35,4 (2014)

8. G. Chen, Y. C. Luo, B. P. Ji and W. su. Journal of food science. 73,6 (2008)

9. R. L. Yang, G. Le, A. Li, J. Zheng, Y. Shi. Nutrition. 22,11 (2006)

10. S. Thangakrishnakumari, A. Nishanthini, S. Muthukumarasamy, V. R. Moha. Bennet (Asclepliadaceae) In Alloxan Induced Diabetic Rats. Asian Journal of Pharmaceutical and Clinical Research, 1,1 (2013)

11. Raghavan P R. Policosanol nanoparticles: U.S. Patent 8,722,093[P]. 2014-5-13.

12. M. Battino, P. Bullon, M. Wilsonand H. Newman. Critical Reviews in Oral Biology \& Medicine. 10,4 (1999) 